\title{
Arqueología histórica en el Perú: la sociedad andina en la TRANSICIÓN ECONÓMICA, POLÍTICA Y SOCIAL
}

\author{
Brendan J. M. Weaver a, Abel Traslaviña ${ }^{b}$, Parker VanValkenburgh ' y Zachary J. Chased
}

\section{Introducción}

Tal y como lo demuestran los artículos recogidos en el primer volumen de esta edición especial, la arqueología histórica del Perú está poblada de preocupaciones metodológicas, temáticas y teóricas que son compartidas por la arqueología andinista y peruana en general. En tal sentido, la arqueología histórica representa una oportunidad de engranar el pasado andino con un presente etnográfico a través del estudio de la cultura material. Por lo tanto, existe una serie de temas comunes que vinculan estos dos volúmenes, tanto a través de las interrogantes metodológicas sobre las intersecciones de datos históricos y arqueológicos, como con aquellas preguntas conceptuales sobre temporalidad e historicidad.

Los artículos de este segundo volumen ofrecen una discusión matizada de las transiciones dentro de la sociedad andina desde el prehispánico hasta el presente, especialmente en los dominios políticos, económicos y sociales —incluyendo lo religioso- - El momento actual de la arqueología histórica en los Andes ofrece a los estudiosos la oportunidad de desarrollar no solo la arqueología andina sino los estudios andinos en general, de una manera que rara vez se ha logrado en otras regiones del mundo. Mientras que en regiones como Norteamérica o Australia hay una fuerte división entre los dominios de la "prehistoria» y la arqueología histórica, causando problemas para los investigadores que trabajan con comunidades indígenas cuya historia atraviesa estos umbrales, tales problemas son menos evidentes en la arqueología histórica desarrollada en América Latina (Zarankin y Salerno 2008; Fowler 2010: 429; Van Buren 2010). La fluidez con la que los arqueólogos de la región han abordado sitios ocupados tanto en períodos prehispánicos como coloniales, ha permitido a los arqueólogos considerar mejor cómo las comunidades indígenas han sido afectadas y a su vez afectan las transformaciones económicas, políticas y sociales locales, regionales y globales. Dada la larga tradición de los arqueólogos andinistas, que tratan de eencontrar un ajuste» con el registro etnohistórico (Julien 1993), especialmente en la arqueología del Horizonte Tardío, parece intuitivo que la arqueología histórica no sea un esfuerzo totalmente aislado — que sea fácilmente subsumida por la arqueología andina más ampliamente definida, contribuyendo a una crítica histórica integral-.

Las transiciones económicas, políticas y sociales en el Perú colonial y republicano ocurrieron en las intersecciones de conexiones globales, mercados y redes de personas, información, ideologías

a Departamento de Historia, Berea College

Correo electrónico: weaverb@berea.edu

${ }^{\text {b }}$ Departamento de Antropología, Vanderbilt University

Correo electrónico: abel.traslavina@vanderbilt.edu

c Departamento de Antropología, Brown University

Correo electrónico: parker_vanvalkenburgh@brown.edu

d Departamento de Antropología, Brigham Young University

Correo electrónico: zachary_chase@byu.edu 
y bienes. Los artículos de este volumen presentan la utilidad de la arqueología histórica como una arqueología dialéctica, enfocada en cómo el presente andino fue creado y transformado por los procesos de un mundo moderno de rápida globalización, y cómo ese mundo moderno fue moldeado por las experiencias coloniales y republicanas peruanas. Nuestros colegas historiadores han examinado exitosamente la organización laboral, los modos de producción andinos, y han trazado las cadenas de suministro y la distribución de bienes, la plata, las plantas y los animales andinos, e incluso las drogas legales e ilegales para los mercados globales (v.g. Topik et al. 2006; Suárez 2009; Crawford 2016). La arqueología histórica andina puede contribuir a una arqueología global que pretende abordar tanto las condiciones locales como los impactos mundiales en la producción, distribución y consumo de la cultura material en el mundo moderno, revelando perspectivas sobre las continuidades culturales andinas, el cambio y la creatividad cultural, y explorar cómo la llegada de nuevos grupos culturales como africanos esclavizados o chinos contratados, ha transformado a la sociedad peruana (Weaver 2016).

Varios de los artículos de este segundo volumen continúan con una exploración de temas transhistóricos — centrándose en las transformaciones sociales afectadas por la incorporación de actores y comunidades y políticas, al Imperio inca y, posteriormente a la colonia española-, especialmente los artículos de Astuhuamán, Chacaltana, Chase, Murphy y Boza, Quilter y Weaver. Un enfoque resaltante de este segundo conjunto de artículos se centra en la importancia de las transformaciones religiosas y en la combinación de las cosmologías andina y cristiana, así como las cosmovisiones mediadas por el imperialismo español. Es importante destacar que estos artículos llaman la atención sobre los procesos coloniales que provocaron estas transformaciones religiosas, específicamente las ideologías de poder que no fueron simplemente impuestas a los pueblos indígenas, sino que se desarrollaron dialécticamente a través de este encuentro colonial. Los artículos de Astuhuamán, Chase, Murphy y Boza, y Quilter, tratan estos aspectos intersectándolos con otros temas importantes relacionados con la evangelización cristiana y la transformación social. En este sentido, el trabajo de Chacaltana es una evaluación íntima del sistema de tambos imperiales en el virreinato, que fue parte integrante de la infraestructura política y económica de la colonia; este artículo también ofrece importantes consideraciones metodológicas y teóricas para analizar el género colonial, la cultura material y el entorno construido.

Los artículos de Weaver y Menaker, ofrecen perspectivas sobre tipos específicos de cultura material como elementos importantes para la construcción de nuevas identidades coloniales andinas, que mezclaron significados españoles e indígenas dentro de una economía global emergente. Por su parte, Jamieson presenta una importante contribución al tema de la escala en la arqueología histórica a través de un análisis de la unidad doméstica, el vecindario, y la parroquia. Junto a Fhon y Coello, Jamieson demuestra que la arqueología histórica andina tiene mucho que aportar a la arqueología del urbanismo. Juntos, estos trabajos analizan diversos momentos de las historias de las ciudades andinas, en particular, el trabajo de Coello trata sobre la creciente secularización de la sociedad peruana en el siglo XIX, llevándonos a pensar en la arqueología histórica más allá de las épocas transconquista y colonial, lo cual también aborda el estudio de la emergencia del estado peruano. La breve discusión que sigue explora la manera en que los arqueólogos están investigando estos temas — la transformación de la sociedad peruana, la religión y la economía política-.

\section{Las sociedades andinas en transición}

Los artículos contenidos en este volumen aportan nuevos datos para las cuestiones más centrales de los estudios andinos, enfocándose en las transformaciones sociales, incluyendo la imposición de una nueva religión y la resistencia, así como el papel de la economía y el poder en los Andes, pero también aportan nuevas perspectivas teóricas y analíticas a estas cuestiones duraderas. Construyen nuevos caminos para unir datos históricos, antropológicos y arqueológicos, y aun cuando se basan en modelos internacionales para el desarrollo de una arqueología histórica interdisciplinaria, permanecen dentro de la gran tradición de los estudios andinos. La producción de 
mercancías y el transporte e intercambio interregionales, fueron centrales para toda la gama de transformaciones sociales de las culturas andinas en la sociedad colonial. Los sitios de producción de nuevos recursos y productos coloniales, fueron a su vez centros de nuevas formas de poder y motores que impulsaron el desarrollo de mercados, que transformaron profundamente a las sociedades andinas. Tales cambios culturales allanaron el camino para las transformaciones en las políticas de identidad, género y relaciones sociales, y se alinearon con ambiciones coloniales de poder y la conquista espiritual de los Andes.

\subsection{Arqueología del poder colonial y cambios religiosos}

Es importante destacar que muchos de los estudios presentados aquí ofrecen un enfoque que combina una preocupación por entender, tanto el proyecto de las conquistas inca y espańola en los Andes, como la remodelación de las economías locales y las estructuras de poder, la conversión religiosa y la cosmología andina en un marco imperial. Estos estudios no solo examinan las transformaciones sociales provocadas por los europeos evangelizadores, sino también las transformaciones producidas a través de las nuevas articulaciones de poder, que se experimentaron a través de nuevas relaciones, tanto en la economía política como en lo espiritual. Algunas aproximaciones históricas anteriores a la conquista religiosa de los Andes han carecido a menudo de una base en las realidades y las prácticas materiales de la experiencia colonial, y rara vez los acercamientos políticos y económicos tomaron seriamente la significación cosmológica del colonialismo, sin embargo en los casos aquí expuestos, desde la arqueología histórica, se toma en cuenta tanto las transformaciones religiosas como sus dimensiones en el poder.

El estudio de Chase sobre la conversión prehispánica y española en Huarochirí, ofrece un enfoque teórico particularmente robusto para examinar estos temas a través de la presentación formal (performativity), centrándose en los conceptos indígenas de la historia y el uso del espacio como punto clave del proyecto de conversión religiosa de los estados inca y español. Como fue mencionado por Chase durante su ponencia, la ventaja de su teoría de performance, es un marco común dentro de lo cual las evidencias arqueológicas e históricas pueden ser consideradas.

El análisis realizado por Astuhuamán sobre la integración política y económica de la región de Piura a los imperios inca y español, se centra en la exploración arqueológica de la iglesia de San Miguel de Piura, la primera iglesia cristiana en el Perú. Los hallazgos del proyecto sugieren que la arquitectura religiosa fue un tema central en el proyecto colonial español en Perú, y la construcción de esa arquitectura institucional fue una de las herramientas más importantes para consolidar el poder e integrar las economías locales. Del mismo modo, Murphy y Boza, a través de la arqueología mortuoria y biológica, ofrecen un enfoque comparativo para comprender las respuestas nativas a la hegemonía española, particularmente en el ámbito de la evangelización. Las autoras reconocen tanto la transformación social, como la continuidad entre las comunidades afectadas por la evangelización cristiana, y ofrecen un resumen de algunos de los primeros datos arqueológicos disponibles para comprender el posible rol continuo e influencia del culto a los ancestros entre las comunidades indígenas. Asimismo, investigan la diversidad de estrategias españolas y nativas, incluyendo la acomodación, el mimetismo, la ambivalencia y la hibridación, algunas de las mismas estrategias y modos de formación de identidad impugnada que Quilter identifica como importantes en la ocupación colonial temprana de Magdalena de Cao Viejo, en el valle de Chicama.

La investigación de Quilter identifica, dentro del registro arqueológico de Magdalena de Cao Viejo, manifestaciones de continuidad cultural y procesos de transformación social marcados por estrategias indígenas de asimilación, acomodación, hibridación y resistencia. ¿̇ qué se refieren los actores nativos en la producción de textiles y otras artesanías? ¿Cómo indexa la cultura material religiosa las específicas prácticas materiales habituales y cambiantes? ¿Qué significa la modificación intencional de piezas de la cultura material española, como los documentos en papel, cortados en diseños geométricos y zoomórficos, y cómo se reflejan las estrategias en estos procesos de transformación social para la comunidad de Magdalena de Cao? 


\subsection{Economía política y la arqueología de la identidad}

Desde fines de la década de 1960 hasta principios de la de 1980, los historiadores (v.g. Assadourian 1979, 1983) y los antropólogos (v.g. Murra 1967-1972, 1978), ofrecieron argumentos paralelos que se centraron en un estudio de las estructuras políticas y económicas andinas en sus propios términos, rompiendo con las preocupaciones y preconcepciones eurocéntricas (Larson 1995: 17). Aunque estos estudiosos representan diversos enfoques teóricos y objetivos para entender a la sociedad andina, se pueden hacer varias comparaciones para los tipos de preguntas centrales que se han desarrollado tanto en los estudios andinos como en los primeros enfoques de la arqueología histórica en el Perú. En el núcleo de estos análisis, estuvo siempre la preocupación por comprender mejor las relaciones entre las comunidades locales y grupos étnicos, y un estado centralizador inca o español.

Siguiendo esta línea cabe destacar trabajos como Trajinantes: Caminos indígenas en la sociedad colonial, siglos XVI/XVII de Luis Miguel Glave (1989), que nos muestra la manera en que el dato interactúa en el espacio a una escala que trasciende lo local, y que permite entender los datos materiales más allá de su sola circunscripción a lugares específicos en las primeras décadas de ocupación peninsular. Glave pone en evidencia una nueva organización política y económica en donde podemos apreciar la complementariedad de los sistemas viarios prehispánicos con los tambos frente a un nuevo mercado, con reglas y dinámicas distintas. De esta manera, se generan nuevos nodos para esta gran red: las ciudades, que transformaron el paisaje económico, político y social, materializado en este nuevo "mercado», que incluyó no solo nuevos productos y tecnologías, sino nuevas formas de hacer negocios en volúmenes y formas nunca antes registrados en tiempos prehispánicos:

Las ciudades fueron también un mercado que completó el explosivo foco de demanda que eran las ciudades de los centros mineros. Su abastecimiento organizó una red de relaciones regionales en sus contornos, pero también éstos se especializaron en la producción de determinados productos agrícolas que se destinaban al consumo extra-regional, cuyo control pasó por función y canal de acumulación de estas ciudades. Los casos más importantes del siglo XVI fueron la coca del Cusco y el vino de Arequipa (incluyendo Moquegua). El destino de fabulosas cantidades de estos productos fue, por un lado, los asentamientos mineros y, por otro, las ciudades. (op. cit.: 37. Énfasis original)

El transporte, a través de las redes coloniales, de bienes y productos vitivinícolas de la costa, coca de las yungas, o textiles del altiplano, a ciudades y centros mineros de la sierra alta, implica una comunicación basada en relaciones mercantiles, y articulada con distintas entidades sociales. En este contexto, las actividades productivas, el manejo de los recursos y su explotación, se insertaron en una nueva dinámica delineada por nuevas tecnologías y que integraron las viejas usanzas comerciales - los productos tradicionales, las caravanas de llameros y la infraestructura prehispánica一, pero que van más allá de aquella escala intra-rural, como la descrita por Ramón (2013) para el caso de los «alfareros golondrinos».

De otro lado, la perspectiva regional ofrecida por el Proyecto Bodegas de Moquegua, bajo la dirección de Prudence Rice, considerado «el primer proyecto multidisciplinario de arqueología histórica en los Andes peruanos» (Rice 2010: 33), ha generado una gran cantidad de datos arqueológicos sobre sitios agroindustriales de producción y centros de mano de obra, lo que ha sido también tratado ampliamente en el volumen anterior.

Es necesario destacar ambos esfuerzos porque inspiraron el diseño de la mesa temática sobre redes de producción en el evento que dio origen a esta publicación, procurando un amplio enfoque que sirva de referencia a futuros estudios. De esta manera, las nuevas investigaciones que aquí se presentan, articulan los sitios de producción y consumo con transformaciones sociales generadas por tales redes de comercio, comunicación y poder.

De acuerdo a lo antes mencionado, consideramos que la cultura material tiene el potencial de revelar patrones específicos de comportamiento, estrategias y procesos, pero sobre todo referenciar múltiples procesos y producción de significados e identidades. Como han sugerido Thomas $\mathrm{H}$. 
Charlton y Patricia Fournier (2011), en su estudio de las primeras cerámicas de barro mexicanas coloniales, las transformaciones en la producción colonial y el uso de la cultura material por las sociedades indígenas americanas, pueden referirse a múltiples procesos sociales incluyendo transformaciones en la política de identidad, la hegemonía colonial, la negociación, la acomodación, la creatividad estética y la economía política. En este sentido, Menaker ofrece un enfoque crítico para ir más allá de las categorías binarias de colonizador/colonizado en el registro arqueológico, a través de un enfoque comparativo en el estudio de un tipo específico de cultura material europea en contextos indígenas durante la época colonial temprana en los Andes españoles: las cuentas de vidrio. A través de estas formas de cultura material, Menaker desarrolla enfoques políticos y económicos, así como una preocupación por la producción del significado, para aproximarnos al cómo una forma de cultura material europea fue insertada en un sistema de valoraciones y prácticas nativas, previamente reservadas para, por ejemplo, las cuentas hechas de Spondylus. Del mismo modo, Weaver examina un conjunto de cultura material estratégicamente empleado por un grupo de trabajadores metalúrgicos especializados, en el Porco español temprano. Estos contextos, muy probablemente relacionados con la figura del yanakuna incaico y compuestos de objetos europeos e indígenas de elite, fueron valorados conjuntamente para hacer referencia a aquellas diferencias sociales. Weaver plantea que la misma categoría de trabajador, sobre la cual descansaba la economía colonial española emergente y las estructuras de poder en los Andes, especialmente aquellas ligadas a la economía minera de Potosí, fue negociada y redefinida por los trabajadores que empleaban estratégicamente tipos específicos de cultura material.

En tal sentido, las estrategias empleadas por los sujetos coloniales para negociar las identidades de raza, clase y género, han sido durante mucho tiempo elementos básicos de la investigación arqueológica histórica (Delle et al. 2000; Orser 2001b). Sin embargo, el género se ha caracterizado a menudo como una de las cuestiones más difíciles de abordar arqueológicamente (Conkey y Spector 1998). En esa línea, es valiosa la contribución de Chacaltana, que centra la lente de la arqueología histórica en la subjetividad colonial de los cuerpos de las mujeres indígenas en el marco del poder colonial y la ideología. De esta manera, cabría preguntarnos ¿Cómo se reconfiguraron los principios andinos de la reciprocidad en la emergente economía política colonial española? Ante ello, Chacaltana explora la transformación del tambo incaico en una nueva institución colonial, examinándolos como nodos que constituyeron las redes políticas y económicas del imperio en los Andes, pero enfocándose específicamente en la regulación de los comportamientos indígenas y la obsesión colonial por los cuerpos de las mujeres indígenas.

\subsection{Espacios domésticos y la arqueología urbana}

El trabajo de Fhon en la casa Bodega y Quadra, ubicada en el centro histórico de Lima, ofrece un estudio de la transformación urbana colonial española. ¿Cómo cambió el uso del espacio urbano colonial a través del tiempo desde principios del siglo XVI hasta mediados del siglo XVIII? ¿Cómo fueron estas transformaciones en la construcción, la arquitectura y el espacio ligadas a los amplios cambios en la evolución social, económica y política de Lima como ciudad cabeza del virreinato? La arqueología histórica llevada a cabo en múltiples escalas y en toda la amplitud de los espacios andinos tiene el potencial de revelar la transformación social andina entre un amplio espectro de la población, desde comunidades rurales andinas, sitios de minería, agricultura o producción artesanal y ciudades cosmopolitas.

En contrapunto al trabajo de Fhon, Jamieson contribuye a este volumen con un análisis de la parroquia de San Blas en Riobamaba (Ecuador). En términos de escala de los contextos arqueológicos, Jamieson hace una importante contribución, teniendo en cuenta el hogar y el barrio como unidades de análisis, en un esfuerzo por comprender las transformaciones sociales de raza y clase social a través de la cultura material. Jamieson, como en el trabajo de Weaver, encuentra que las ocupaciones particulares hicieron uso de clases específicas de cultura material para asignarles estatus. 
En este punto, podemos ver cómo los contextos urbanos en los Andes muchas veces ofrecen al investigador sitios ricamente estratificados para explorar sitios domésticos, productivos, comerciales y religiosos a través de la longue durée. Aun cuando la mayoría de los artículos de este volumen muestran el potencial de la arqueología histórica para aproximarnos a la comprensión del cambio político, económico y social en el contexto del colonialismo español en el Perú virreinal, es necesario señalar que la arqueología histórica no es solamente la arqueología colonial. Como subdisciplina limitada metodológica y temáticamente, debido a las disciplinas de la historia, la antropología y la arqueología, aborda eventos, procesos y actores (Deagan 1988; Mrozowski 1988; Deetz 1989; Orser 2001a), y, como lo demuestran los artículos de este volumen, hace uso de la cultura material y la evidencia histórica para comprender el cambio social y la continuidad entre las épocas prehispánica, colonial y republicana. En tal sentido, el artículo de Coello aborda la arqueología urbana como la arqueología del urbanismo, trazando transformaciones en los patrones de la vida urbana del siglo XIX, ofreciendo evidencia material y arquitectónica para entender las transiciones de la vida religiosa colonial a la aparición del secularismo republicano. De esta manera Coello muestra que es posible ampliar el rango de acción de la arqueología histórica peruana, así como su potencial, para también considerar los procesos más recientes que dieron origen a nuestra sociedad moderna, de manera continua, desde sus legados coloniales.

\section{Contribuciones de la arqueología histórica peruanista a la arqueología mundial}

Desde el Precerámico hasta la arqueología de la modernidad, la arqueología andinista ya ha contribuido ampliamente a la arqueología comparativa global. Por ejemplo, los estudios de las tempranas formaciones políticas complejas de la región andina han ofrecido una crítica de las teorías clásicas del Estado y su formación (v.g. Collier et al. 1982; Haas et al. 1987; Lumbreras 1972, 1988, 1994; Moseley y Cordy-Collins 1990; Netherly 1993; Stanish 2001; Yoffee 2005). De la misma manera, la arqueología histórica peruanista tiene mucho que aportar a la comprensión arqueológica de la formación de identidades, el conflicto, la fragmentación política, y transformaciones económicas y políticas, entre una variedad de temas. Específicamente, la arqueología histórica, como una disciplina dialéctica y centrada internacionalmente —una arqueología del mundo moderno - (véase Orser 1996), tiene la potencialidad para mejor entender los orígenes de las instituciones más importantes de la modernidad, a través de un enfoque en la gobernanza moderna temprana, el crecimiento del sistema mundial capitalista, la formación del estado-nación y la racialización de sujetos coloniales.

Los artículos de este volumen muestran cómo la naturaleza interdisciplinaria de la arqueología histórica está excepcionalmente posicionada, para ofrecer una nueva perspectiva sobre cuestiones y temas que han sido durante mucho tiempo, ámbito de los estudios andinos, tradicionalmente del dominio de la historia, la antropología, o la arqueología precolombina. A medida que aumente el interés por la arqueología histórica en Perú, será relevante para su propio desarrollo y el de los estudios andinos, pero también con miras a desarrollar aquel potencial en hacer contribuciones importantes más allá de la región andina. De esta manera, consideramos que la arqueología histórica puede jugar el rol no solo de contribuir a una mejor comprensión del lugar que ocupa el Perú en el mundo moderno, sino además de contribuir a una mejor comprensión del desarrollo del mundo Atlántico y la modernidad de cara al Pacífico. 


\section{REFERENCIAS}

Assadourian, C. S.

1979 La producción de la mercancía dinero en la formación del mercado interno colonial: El caso del espacio peruano, siglo XVI, en: E. Florescano (ed.), Ensayos sobre el desarrollo económico de México y América Latina (1500-1975), 223-292, Fondo de Cultura Económica, México.

1983 Dominio colonial y señores étnicos en el espacio andino, Revista Latinoamericana de Historia Económica y Social 1, 7-20.

\section{Charlton, T. H. y P. Fournier}

2011 Pots and plots: The multiple roles of early colonial red wares in the Basin of Mexico (Identity, resistance, negotiation, accommodation, aesthetic creativity, or just plain economics?), en: M. Liebmann y M.S. Murphy (eds.), Enduring conquests: Rethinking the archaeology of resistance to Spanish colonialism in the Americas, 127-148, School for Advanced Research Press, Santa Fe.

Collier, G. A., R. Rosaldo y J. D. Wirth (eds.)

1982 The Inca and Aztec states, 1400-1800: Anthropology and history, Academic Press, New York.

\section{Conkey, M. W. y J. D. Spector}

1998 Archaeology and the study of gender, en: K. Hays-Gilpin y D. S. Whitley (eds.), Reader in gender archaeology, 11-46, Routledge, London.

\section{Crawford, M.J.}

2016 The Andean wonder drug: Cinchona bark and imperial science in the Spanish Atlantic, 1630-1800, The University of Pittsburgh Press, Pittsburgh.

\section{Deagan, K.}

1988 Neither history nor prehistory: Questions that count in historical archaeology, Historical Archaeology 22(1), 7-12. https://doi.org/10.1007/BF03374494

Deetz, J.

1989 Foreword, en: R. E. Meyer (ed.), Cemeteries and Gravemarkers: Voices of American Culture, ix-xiv, UMI Research Press, Ann Arbor.

Delle, J. A., S. A. Mrozowski y R. Paynter (eds.)

2000 Lines that divide: Historical archaeologies of race, class, and gender, The University of Tennessee Press, Knoxville.

Fowler, W. R., Jr.

2010 Historical archaeology in Yucatan and Central America, en T. Majewski y D. Gaimster (eds.), International handbook of historical archaeology, 429-447, Springer, New York. https://doi.org/10.1007/978-0-38772071-5_25

Glave, L. M.

1989 Trajinantes: caminos indigenas en la sociedad colonial: siglos XVI-XVII, Serie Tiempo de Historia, Instituto de Apoyo Agrario, Lima.

Haas, J., S. Pozorski y T. Pozorski (eds.)

1987 The origins and development of the Andean state, Cambridge University Press, Cambridge.

Julien, C. J.

1993 Finding a fit: Archaeology and ethnohistory of the Incas, en: M. A. Malpass (ed.), Provincial Inca: Archaeological and ethnohistorical assessment of the impact of the Inca state, 177-233, University of Iowa Press, Iowa City.

\section{Larson, B.}

1995 Andean communities, political cultures, and markets: The changing contours of a field, en B. Larson, O. Harris y E. Tandeter (eds.), Ethnicity, markets, and migration in the Andes: At the crossroads of history and anthropology, 5-53, Duke University Press, Durham. https://doi.org/10.1215/9780822379867-001

Lumbreras, L. G.

1972 De los orígenes del estado en el Perú: Nueva crónica sobre el viejo Perú, Milla Batres, Lima.

1988 El estudio arqueológico del estado, Gaceta Arqueológica Andina 4(16), 3-5. 
1994 Acerca de la aparición del Estado, Boletín de Antropología Americana 29, 5-33. Recuperado de: http:// www.jstor.org/stable/40978062

Mrozowski, $S$.

1988 Historical archaeology as anthropology, Historical Archaeology 22 (1), 18-24. https://doi.org/10.1007/ BF03374496

Moseley, M. y A. Cordy-Collins (eds.)

1990 The northern dynasties: Kingship and statecraft in Chimor, Dumbarton Oaks Research Library and Collection, Washington, D.C.

Murra, J. V.

1967- El «control vertical» de un máximo de pisos ecológicos en las economías de las sociedades andinas, en: I.

1972 Ortíz de Zúniga (ed.), Visita a la provincia de León de Huánuco [1562], 2 vols., 429-476, Universidad Nacional Hermilio Valdizán, Huánuco.

1978 La organización económica del Estado inca, Siglo Veintiuno, México.

\section{Netherly, P. J.}

1993 The nature of the Andean state, en: J. S. Henderson y P. J. Netherly, (eds.), Configurations of power, 11-35, Cornell University Press, Ithaca, N.Y.

Orser, C. E., Jr.

1996 A Historical Archaeology of the Modern World, Plenum Press, New York.

2001a The anthropology in American historical archaeology, American Anthropologist, New Series 103(4), 621633. https://doi.org/10.1525/aa.2001.103.3.621

Orser, C. E., Jr. (ed.)

$2001 \mathrm{~b}$ Race and the archaeology of identity, The University of Utah Press, Salt Lake City.

Ramón Joffre, G.

2013 Los alfareros golondrinos. Productores itinerantes en los Andes, Instituto Francés de Estudios Andinos/ Sequilao Editores, Lima.

Rice, P. M.

2010 La industria vitivinícola colonial de Moquegua, Perú, Estudios Avanzados 1(14), 29-62. Disponible en: $<$ http://www.cecta.cl/ojs/index.php/ideas/article/view/4>.

Stanish, C.

2001 The origin of state societies in South America, Annual Review of Anthropology 30, 41-64. https://doi. org/10.1146/annurev.anthro.30.1.41

Suárez Espinoza, M.

2009 El Perú en el mundo atlántico (1520-1739). Compendio de historia económica del Perú. Economía del Periodo Colonial Temprano, Banco Central de Reserva del Perú/Instituto de Estudios Peruanos 2, 229311, Lima.

Topik, S., C. Marichal y Z. Frank (eds.)

2006 From silver to cocaine: Latin American commodity chains and the building of the world economy, 1500-2000, Duke University Press, Durham.

Van Buren, M.

2010 The archaeological study of Spanish colonialism in the Americas, Journal of Archaeological Research 18 (2), 151-201. https://doi.org/10.1007/s10814-009-9036-8

Weaver, B. J. M.

2016 Perspectivas para el desarrollo de una arqueología de la diáspora africana en el Perú: Resultados preliminares del Proyecto Arqueológico Haciendas de Nasca, Allpanchis 80 (2do semestre, 2012), 85-120.

Yoffee, N.

2005 Myths of the archaic state: Evolution of the earliest cities, states, and civilizations, Cambridge University Press, Cambridge. https://doi.org/10.1017/CBO9780511489662

Zarankin, A. y M. A. Salerno

2008 Looking South»: Historical archaeology in South America, Historical Archaeology 42 (2), 38-58. https:// doi.org/10.1007/BF03377153 